\title{
Association Between Foot Posture and Tibiofemoral Contact Forces During Barefoot Walking in Patients with Knee Osteoarthritis
}

Takanari Kubo ( $\sim$ b8987253@kio.ac.jp )

Kio University

Daisuke Uritani

Kio University

Shinya Ogaya

Saitama Prefectural University

Syunsuke Kita

Saitama Prefectural University

Takahiko Fukumoto

Kio University

Tadashi Fujii

Kashiba Asahigaoka Hospital

Yusuke Inagaki

Kashiba Asahigaoka Hospital

Yasuhito Tanaka

Nara Medical University

Hidetaka Imagita

Kio University

\section{Research Article}

Keywords: Tibiofemoral contact force, Foot, Walking, Musculoskeletal model

Posted Date: January 17th, 2022

DOI: https://doi.org/10.21203/rs.3.rs-1213732/v1

License: (c) (1) This work is licensed under a Creative Commons Attribution 4.0 International License.

Read Full License 


\section{Abstract \\ Background}

Accumulating evidence indicates that abnormal foot posture and function are risk factors for knee osteoarthritis (OA). However, the relationship between foot posture and function and tibiofemoral contact force (CF) during habitual weight-bearing activities remains unclear. This study aimed to determine the association between $\mathrm{CF}$ and foot posture and function while walking.

\section{Methods}

In total, 18 patients with knee $\mathrm{OA}$ and 18 healthy individuals participated in this cross-sectional study. Foot parameters were evaluated by foot Posture Index (FPI), Staheli Arch Index (SAI), hallux valgus angle, calcaneus inverted angle relative to the floor as a static rearfoot posture, navicular height, and toe grip strength. In addition, all participants underwent kinetic and kinematic measurements during a selfselected speed gait. The measurement device used was the three-dimensional motion analysis system with a sampling rate of $120 \mathrm{~Hz}$. The musculoskeletal model, which has $92 \mathrm{Hill}$-type muscle-tendon units with 23 degrees of freedom, was used to calculate CF. Pearson's correlation coefficient was used to investigate the association between foot parameters and total, medial, and lateral CF of the first and second peaks.

\section{Results}

A significant negative correlation was observed between Walking SAI and first medial CF in control participants $(r=-0.515, p=0.029)$. SAI was also significantly positively correlated with first medial CF in patients with knee OA $(r=0.488, p=0.040)$.

\section{Conclusions}

Our findings reveal a correlation between the medial first peak CF and the SAl. This study indicates that people with knee $\mathrm{OA}$ and flatfoot have excessive first medial CF during walking.

\section{Background}

Knee osteoarthritis (KOA), a highly prevalent disease in older adults, leads to chronic pain, stiffness, and disability. Various factors contribute toward dynamic mechanical loading of the knee joint during walking that can lead to joint pain [1], cartilage damage [2], and bone deformities [3]. Further, the development and progression of KOA is affected by excessive rotational and/or contact loading [2,3]. Although radiographic KOA is rare in Japanese people aged $<40$ years, its prevalence among adults aged $>40$ years has been demonstrated to be $42.6 \%$ in men and $62.4 \%$ in women [4]. 
Many studies have assessed the association between tibiofemoral joint (TFJ) contact loading by knee adduction moment (KAM) and knee function or alignment. For example, Winby et al. [5] demonstrated that KAM during walking was influenced by knee-spanning muscle contractions in healthy individuals, and Sharma et al. [6] found a negative association between KAM during walking and joint space width in KOA patients. However, limited studies have investigated the relationship between foot posture and function and mechanical loads on the knee joint. Increased rearfoot eversion, rearfoot internal rotation, and forefoot inversion are associated with reduced KAM during walking [7], and accumulating evidence indicates that KOA patients have abnormal foot posture and foot function, such as hallux valgus [8], flatfoot [9], and decreased toe grip strength (TGS) [10]. Notably, Gross et al. [9] found a significant association between flatfoot measured by footprint data and cartilage damage of the TFJ.

In clinical practice, foot evaluation is difficult using a three-dimensional (3D) motion analysis device (e.g., the Oxford Foot Model) owing to the time and cost involved. Many patients prefer to undergo physiotherapy and insole insertion after static foot evaluation that can be easily performed in clinical practice; hence, its relationship with TFJ loads must be determined. Notably, many methods for static foot evaluation in clinical practice are devoid of radiographic measures. Rearfoot angle (RFA), navicular drop, and footprint as a uniplanar assessment of foot posture are often used [11]. In contrast, the Foot Posture Index is a multi-planar tool that has gained popularity recently.

Many studies have used KAM as a surrogate marker of TFJ contact loading during activities, such as walking [6]. KAM is based on inverse dynamics, which only accounts for external parameters, i.e., ground reaction forces (GRF) and joint angles. Musculoskeletal modeling enables the calculation of muscle forces (MF) and joint contact forces and gives insights into the internal loading of body [5]. Notably, Lerner et al. [12] developed a model in OpenSim to accurately assess medial and lateral tibiofemoral contact force (CF) in a tibiofemoral implant study. This model uses subject-specific knee joint medial and lateral compartment contact locations and joint alignment to more accurately estimate medial and lateral CF (MCF, LCF). Moreover, a systematic review investigating the correlation between CFs and external joint moments found that the MCF and total CF (TCF) in the first half of stance can be predicted using external joint moments. However, worse correlations were found for the peak in the second half of stance and lateral CF [13]. Therefore, while investigating the second peak in the stance phase and lateral knee joint loading, it is better to calculate CF using musculoskeletal models.

Despite these findings, the biomechanical relationship between foot posture and function and CF remains unclear. Therefore, this study aimed to clarify the association between CF and foot posture and function during walking in KOA patients. We hypothesized that participants with abnormal foot posture and function, such as excessive flatfoot, hallux valgus, and lower TGS, have increased CF during walking.

\section{Methods}

\section{Participants}


Eighteen patients with medial KOA (4 men and 14 women; mean age: $60.17 \pm 6.96$ years) were recruited from the outpatient department of orthopedics at Kashiba Asahigaoka Hospital; 18 healthy adults (4 men and 14 women; mean age: $62.78 \pm 8.58$ years) were recruited from participants in a health promotion project conducted by Koryo Town, Nara, Japan, and from the staff members of Kashiba Asahigaoka Hospital. Participant characteristics are shown in Table 1.

Table 1

Participant characteristics

\begin{tabular}{|llll|}
\hline & Controls $(\mathbf{n = 1 8})$ & KOA (n=18) & p-value \\
\hline Age (years) & $62.78(8.58)$ & $60.17(6.96)$ & 0.323 \\
\hline Height $(\mathrm{cm})$ & $157.83(8.89)$ & $161.44(7.38)$ & 0.194 \\
\hline Weight $(\mathrm{kg})$ & $54.33(10.60)$ & $65.97(11.07)$ & $0.003^{*}$ \\
\hline Female, $\mathrm{n}(\%)$ & $14(78 \%)$ & $14(78 \%)$ & 1.000 \\
\hline Walking Speed (m/s) & $1.30(0.15)$ & $1.18(0.13)$ & $0.016^{*}$ \\
\hline Tibiofemoral alignment (deg) & $177.35(1.95)$ & $180.28(1.87)$ & \\
\hline K/L grade, 2/3/4 & N/A & $11 / 7 / 0$ & \\
\hline $\begin{array}{l}\text { K/L grade, Kellgren and Lawrence grade. Values are presented as mean (SD). P-values were obtained } \\
\text { from the independent t-test. *P<0.05 }\end{array}$ & & & \\
\hline
\end{tabular}

The inclusion criteria for the participants with medial KOA were as follows: Age $>45$ years and radiographic changes with Kellgren-Lawrence $(\mathrm{KL})$ grade of 2 or higher in the medial tibiofemoral compartment. The exclusion criteria for the participants with medial KOA were as follows: A history of any other orthopedic injury in the lower extremities, neurological injury, rheumatoid arthritis, joint surgery in lower extremities, lateral KOA, or use of an assistive device. For control participants, the inclusion criteria were as follows: age $>45$ years, walking independently without any ambulatory assistive device, and no pain in the knee joint, and we excluded those with any previous surgical treatment for the lower limbs or trunk or known neuromuscular or musculoskeletal pathologies.

\section{Gait analysis}

Six VICON MX-F20 cameras (Vicon Metrics, Oxford, UK) and four floor-embedded force platforms $(2 \times$ 9281B, Kistler Instrument Corporation, Switzerland; $2 \times$ AMTI BP400600, Advanced Mechanical Technology Inc., USA) were used to measure 3D kinematics and GRF with a sampling rate of $120 \mathrm{~Hz}$, respectively. The plug-in-gait full body 39 markers set was used. These markers were attached to each participant's skin at the following anatomical landmarks: forehead, back of the head, shoulder, $7^{\text {th }}$ cervical vertebra (C7), $10^{\text {th }}$ thoracic vertebra, upper arm, elbow, forearm, wrist, finger, right back, anterior superior iliac spines (ASIS), posterior superior iliac spines (PSIS), thigh, lateral knee, tibia, lateral ankles, heels, and second metatarsal heads. 
Participants were asked to walk at a self-selected speed, barefoot, along a flat 8-m walkway. Three successful walking trials were recorded. Stance phases during gait were determined using vertical GRF data. GRFs were low-pass filtered using $10-\mathrm{Hz}$ cut-off frequencies for walking. The threshold value for the stance phase was a GRF of $25 \mathrm{~N}$ or more in the vertical direction. The walking speed was calculated from the distance of the right and left second metatarsal head marker positions during 6 steps and the time taken.

\section{Musculoskeletal model}

A musculoskeletal model with 18 body segments and 92 muscle-tendon actuators in OpenSim was used to compute the CF $[12,14]$. In this knee mechanism, the medial and lateral TFJs share all forces transmitted between the femur and tibia and resolve them as the medial and lateral CFs required to balance the net reaction forces and frontal-plane moments across the TFJ. Correspondingly, the knee remains a single degree of freedom (DOF) joint with motion only in the sagittal plane. Therefore, the model includes a spline function on the knee axis, which takes into account the anterior-posterior translations $[12,14]$. The model has only a single DOF for dorsi-/plantarflexion and a single axis for the ankle joint. For each participant, subject-specific models were created based on the approach reported by Lerner et al. [12]. In the KOA group, standing anatomical motion capture markers were supplemented with anteroposterior weight-bearing radiograph of the lower-extremity $[12,15]$. The dimensions of each body segment in the model were scaled based on relative distances between pairs of markers obtained from a motion-capture system and the corresponding virtual marker locations in the model. Next, we modified each participant's scaled model and created a subject-specific model by specifying their lower extremity alignment from the radiographs in KOA patients. An anteroposterior radiograph of the participant's lowerextremity was used to determine the subject-specific alignment for the musculoskeletal model. The tibiofemoral alignment was found by drawing lines connecting the hip, knee, and ankle joint centers, which were defined as the center of the femoral head, center of the femoral condyles, and midpoint of the medial and lateral margins of the ankle, respectively. The tibiofemoral alignment was adjusted only for the healthy adults using the marker information [16]. The model was then used to calculate joint angles and moments during walking trials using OpenSim inverse kinematics and inverse dynamics, respectively. MF were estimated using static optimization. Mini-mizing of activation squared was used as the static optimization criterion for the cal-culation of MF. These results were used to calculate the joint reaction force (JRF) in OpenSim.

\section{Foot assessment}

The FPI was calculated in a relaxed standing position to assess foot posture based on a six-item standard protocol [17]. The FPI included the following: 1) talar head palpation, 2) supra and infra lateral malleolar curvature, 3) calcaneal frontal plane position, 4) prominence in the region of the talonavicular joint, 5) congruence of the medial longitudinal arch, and 6) abduction/adduction of the forefoot on the rear foot. Each item was scored on a scale of $-2,-1,0,+1$, and +2 ( 0 for neutral, -2 for clear signs of supination, and +2 for clear signs of pronation) [17]. The total score ranged from -12 to +12 , with a larger 
positive value indicating a more pronated foot. For foot type identification, normative values with scores of $\geq 6$ represent a pronated foot type; those with scores of $0-5$ represent a neutral foot; and those with scores of $\leq-1$ represent a supinated foot [17]. The inter-rater and intra-rater reliability for measuring FPI was excellent (ICC: 0.98 and 0.91 , respectively).

A force platform (WinFDM; Zebris, Isny im Allgäu, Germany) was used to measure static and dynamic footprint data. Static footprint data were recorded for 10 seconds with a participant standing on the platform with double limb support. Dynamic footprint data were collected during walking at a selfselected speed on the platform. The Staheli Arch Index (SAI), the ratio of the smallest width of the midfoot to the greatest width of the rearfoot [18], was calculated from footprint data. The value of the SAI increases with increasingly planus foot morphology and takes on a value of zero with cavus foot morphology. The widths were measured by ImageJ software [19]. A previous validity study indicated a moderately significant correlation between SAI measurements of foot morphology and radiological measurements of total foot morphology [18]. The average value of two measurements was used in the analysis. The reliability of SAI measurements using ImageJ was calculated by 2 testers in 20 participants. The inter-rater and intra-rater reliability for measuring the SAI was excellent (ICC: 0.96 and 0.91 , respectively).

The hallux valgus angle (HVA) and calcaneus inverted angle relative to the floor (CIA) were measured using a goniometer. HVA was the angle formed by the first metatarsal bone and the proximal phalanx of the hallux [20]. Janssen et al. [20] investigated the reliability and concurrent validity of universal goniometer measurements of the HVA compared to those of radiographs and found a good intraclass correlation coefficient. CIA was defined as the angle formed by the bottom of the calcaneal tuberosity and the enthesis of the Achilles tendon [21]. The reliability of CIA measurements was calculated by 2 testers for 20 participants. The inter-rater and intra-rater reliability for measuring the CIA was good (ICC: 0.92 and 0.86 , respectively).

Navicular height $(\mathrm{NH})$ /foot length was measured as the length from the floor to the top of the navicular tuberosity [22]. The measure of $\mathrm{NH}$ in a standing or sitting position demonstrated good intra- and interrater reliability [22].

Navicular drop test (ND-t) was recorded as the difference in $\mathrm{NH}$ between relaxed standing and sitting position [21]. ND-t had moderate intra- and inter-rater reliability [22].

TGS was measured using a toe grip dynamometer (T.K.K.3362; Takei Scientific Instruments Co., Ltd., Niigata, Japan) with participants sitting upright [23]. Participants gripped the grip bar with maximal effort for about 3 seconds. The average value of two measurements was used in the analysis. In this measurement protocol, substantial to almost perfect inter- and intra-rater reliability was found in people aged $60-79$ years [23].

\section{Statistical analysis}


Gait trial data were normalized by time to $0 \%-100 \%$ of the stance phase in the gait cycle. Each $\mathrm{CF}$, normalized by body weight (BW), was represented as a time series throughout the stance phase (SP). The first and second peaks of the TCF, MCF, and LCF were identified. The maximum CF during $0 \%-50 \%$ of SP was defined as the first peak, and the maximum CF during $51 \%-100 \%$ of SP was defined as the second peak.

Differences in demographic data, CF, FPI, Standing SAI, Walking SAI, HVA, CIA, NH, ND-t, TGS, and gait speed between the groups were evaluated by a t-test. Sex differences were examined by chi-square test. For the relationship between foot posture or function and CF, correlation coefficients of CF with foot parameters that were significantly different between the groups were calculated. If a significant correlation between CF and a foot parameter was found for the first peak, we then additionally performed a correlation between the MF at the peak and the foot parameter. The correlation between walking speed and CF or foot parameters was further calculated. The Shapiro-Wilk test was used to determine the normal distributions. In normally distributed data, Pearson's correlation tests were used. The power analysis was performed by selecting a desired power of 0.8 .

All statistical analyses were performed using SPSS Statistics for Windows, version 22.0 (IBM Corp, Tokyo, Japan). A p-value of $<0.05$ was considered significant.

\section{Results}

There were no significant differences in age, height, and sex between KOA patients and controls (Table 1). The participant weight was significantly higher in the KOA patient group than in the control group. KOA patients walked significantly slower than the controls. Foot assessment revealed no significant differences in the FPI, HVA, CIA, NH, and TGS between KOA patients and controls (Table 2). The Standing SAl, Walking SAl, and ND-t were significantly higher in KOA patients than in controls. Figure 1 shows the experimental results for the total $\mathrm{CF}$ and medial and lateral CF values. No differences were detected in the first and second peak medial, lateral, and total CF values (Table 3). Figure 2 shows the experimental results for the MF of the knee-spanning muscle values. These are similar to the waveforms in previous studies [5]. None of the parameters correlated with walking speed. Power analysis was performed to measure the minimum number of samples required to detect differences in foot parameters between the control and KOA groups. Post-hoc power analysis based on Walking SAI, Standing SAI, and ND-t indicated that a minimum of 33,17 , and 36 participants, respectively, would be needed per group to detect a significant difference at a power $>0.8$ and $a=0.05$. 
Table 2

Foot characteristics

\begin{tabular}{|c|c|c|c|}
\hline & Controls $(n=18)$ & KOA $(n=18)$ & p-value \\
\hline FPI (point) & $2.11(2.42)$ & $2.56(2.91)$ & 0.622 \\
\hline Standing SAI (mm/mm) & $0.31(0.26)$ & $0.56(0.24)$ & $0.005^{\star \star}$ \\
\hline Walking SAI (mm/mm) & $0.46(0.23)$ & $0.62(0.17)$ & $0.020^{\star}$ \\
\hline HVA (deg) & $17.22(7.36)$ & $13.56(7.14)$ & 0.138 \\
\hline CIA (deg) & $3.72(2.19)$ & $4.28(3.69)$ & 0.587 \\
\hline $\mathrm{NH}(\mathrm{mm} / \mathrm{mm})$ & $0.15(0.02)$ & $0.15(0.02)$ & 0.616 \\
\hline ND-t (mm) & $0.46(0.37)$ & $0.72(0.37)$ & $0.042^{*}$ \\
\hline TGS (kg) & $12.17(4.06)$ & $12.93(5.09)$ & 0.627 \\
\hline
\end{tabular}

Table 3

Peak values of the first and second CFs

\section{Controls $(n=18)$}

KOA $(n=18)$

p-value

Total CF

$\begin{array}{llll}\text { First Peak (BW) } & 3.51(0.91) & 3.45(0.87) & 0.825 \\ \text { Second Peak (BW) } & 3.61(1.37) & 4.17(1.11) & 0.188\end{array}$

\section{Medial CF}

$\begin{array}{llll}\text { First Peak (BW) } & 2.71(0.78) & 2.65(0.45) & 0.776 \\ \text { Second Peak (BW) } & 2.58(0.90) & 3.09(0.83) & 0.087\end{array}$

\section{Lateral CF}

$\begin{array}{llll}\text { First Peak (BW) } & 1.24(0.41) & 1.60(0.71) & 0.070 \\ \text { Second Peak (BW) } & 1.79(0.60) & 1.43(0.47) & 0.052\end{array}$

All data are expressed as mean (SD). CF, Contact force. P-values were obtained from independent ttest. ${ }^{* \star} \mathrm{p}<0.01,{ }^{*} \mathrm{p}<0.05$

According to Pearson's correlation coefficient, the first peak MCF was significantly negatively correlated with SAI during walking $(r,-0.515 ; p, 0.029 ; 95 \% \mathrm{Cl}:-0.792$ to -0.064$)$ in KOA patients. However, in healthy adults, the first peak MCF was significantly positively correlated with SAl during walking (r, 0.488; $p$, 0.040; 95\% Cl: 0.027-0.778) (Figure 3). 
No significant correlation was found between the first and second peak values of the CF and Standing SAl and ND-t. No significant correlation was found between MF and SAI.

\section{Discussion}

To our best knowledge, this study is the first to investigate the relationship between CF calculated by musculoskeletal model and foot posture and function. Our results are compliant with those of previous musculoskeletal model-based studies [5, 24].

Peak TCF of about 3-4 times BW was calculated. CFs showed no statistically significant differences between the groups in terms of first and second peaks. This result is consistent with that of a similar previous study [25]. Merireles et al. [25] reported that CFs in patients with early KOA (KL $\leq 2)$ were not significantly different from those in healthy adults. Although the magnitudes of first and second peaks in the KOA group in this study have different trends compared to those in the study by Merireles et al. [25], the majority of patients had $\mathrm{KL} \leq 2$, and the relative peaks of $\mathrm{CFs}$ between the groups were nonsignificant.

Correlations between the first peak MCF and the SAI in controls and KOA patients were found. However, MF showed no significant correlation with SAI at the time of the first peak MCF. Therefore, MF had almost no effect on the first peak MCF due to the foot abnormality of the medial arch evaluated by SAI. We could not draw a direct relationship as there is no muscle that extends from the knee joint to the foot.

Our results showed correlations between the first peak MCF and SAI during walking in controls and KOA patients, which were attributed to the shock-absorbing function of the truss that is formed by the calcaneus, midtarsal joint, and metatarsals [26] and functions with the supinated foot position at heel strike during walking. Thereafter, the subtalar joint immediately pronates when transitioning from heel strike to loading response phase. Pronation of the foot absorbs GRF [26], and the truss sinks to absorb shock in that phase. Notably, in people with flatfoot or cavus foot, the truss does not function, thereby resulting in excessive CF.

Our results showed that the correlations between the first peak MCF and Walking SAI were negative in KOA patients and positive in healthy controls. In healthy and KOA participants, only the MCF was associated with SAI, but the LCF was not significantly associated with SAI, probably due to the GRF and knee joint position. KAM contributes to the $\mathrm{CFs}$. The moment arm is determined by the distance of GRF from the center of the knee joint. The position of the calcaneus during walking in healthy participants is different from that in patients with foot problems. The lateral shift of the calcaneus during walking in children with flatfoot results in a low first peak KAM because of the point of GRF application due to the calcaneal position [27]. In fact, shoe modifications produce a lateral shift in the point of GRF and reduce the first peak KAM in healthy adults [28]. Therefore, flat feet in healthy adults may bring the GRF closer to the center of the knee joint and have the potential to indirectly decrease CF. In this study, it is believed that among the CFs, only the MCFs, which are most affected by the length of the moment arm, have extracted correlations. However, based on the present results alone, knee joint alignment as a factor for the difference in results cannot be excluded. 
KOA patients achieve a relatively flatter position of the foot while walking as compared to healthy adults. Further, previous research has shown that people with flatfoot show a high peak pressure for the medial foot area during walking [29]. In people with flatfoot, the center of pressure may be different during medial shift of the foot; as a result, the KAM moment arm is expected to increase. Moreover, the range of motion of the rearfoot is smaller in KOA patients than in healthy participants [30]. Therefore, the lateral shift of the calcaneus is unlikely to occur in KOA patients.

There was a significant difference in ND-t, Standing SAl, and Walking SAI between the healthy and KOA groups. During most weight-bearing activities, the posture and motion of the foot and knee are coupled within a closed kinematic chain, such as the movements in the horizontal plane and frontal plane [30]. However, despite the fact that Walking SAI is correlated, ND-t and Standing SAI had no correlation with CF during walking. Controversies regarding static foot alignment reflecting a dynamic foot morphology exist $[31,32]$. Additionally, the dynamic foot motion during walking in KOA patients differ from that in healthy adults [30]. Therefore, it is highly possible that the SAl evaluated during walking represented the CF during walking. Furthermore, closed chain coupling may link excessively planus foot morphology to excessive internal rotation of the lower extremity [33]. However, in this study, only CF was used, and foot, ankle, knee, or hip kinematics and rotational stress were not investigated.

This study had some limitations. First, the number of males was fewer than that of females. Future studies will include an equal number of male and female participants. Additionally, future studies should increase the number of participants. Second, the model had only a single DOF for flexion/extension and a single axis for the knee joint. Therefore, internal/external rotation during knee joint movement could not be considered. Third, the model of the ankle joint and midfoot motion used herein was for dorsiflexion and plantarflexion only. Therefore, this model did not accurately reflect the movement of the coronal plane motion in the subtalar and metatarsophalangeal joints. However, the GRF was actually assessed in this study; its relationship with the position of the center of gravity or knee joint was clarified using marker trajectory data. Furthermore, gait speed in patients with knee OA was much slower than that in controls. Thus, different results may be obtained in patients with knee OA who maintain gait speed. Finally, further studies are needed to investigate detailed relationships using more discriminating foot morphology indexes. Future studies directed toward investigating patients diagnosed with pathological foot positions or functions are warranted.

\section{Conclusions}

The first peak medial CF was significantly correlated with the SAI. The findings reveal the relationship between cavus foot and CF during walking in patients with knee OA. This study suggests that patients with knee $O A$ and flatfoot have excessive first MCF during walking.

\section{List Of Abbreviations}


3D, three-dimensional; ASIS, anterior superior iliac spines; BW, body weight; CF, contact force; CIA, calcaneus inverted angle; DOF, degree of freedom; FPI, foot Posture Index; GRF, ground reaction forces; HVA, hallux valgus angle; KAM, knee adduction moment; KL, Kellgren-Lawrence; KOA, knee osteoarthritis; MF, muscle forces; NH, navicular height; ND-t, navicular drop test; PSIS, posterior superior iliac spines; RFA, Rearfoot angle; SAJ, Staheli Arch Index; SP, stance phase; TFI, tibiofemoral joint; TGS, toe grip strength.

\section{Declarations}

\section{Ethics approval and consent to participate}

The study protocol was approved by the institutional review boards of Kashiba Asahigaoka Hospital (201911001) and Kio University (H30-42). All participants provided written informed consent. This study was performed in accordance with the Declaration of Helsinki.

\section{Consent for publication}

Not applicable.

\section{Availability of data and materials}

The datasets used and analyzed during the current study available from the corresponding author on reasonable request.

\section{Competing interests}

The authors declare that they have no competing interests.

\section{Funding}

This study was supported by JSPS KAKENHI (Grant number, 19K11403; http://www. jsps.go.jp/jgrantsinaid/index.html).

\section{Authors' contributions}

All authors have made substantial contributions to (1) the conception and design of the study, or acquisition of data, or analysis and interpretation of data, (2) drafting the article or revising it critically for important intellectual content, (3) final approval of the version to be submitted. The specific contributions in this article of the authors are as following: (1) Conception and design of the study: TK, DU, SO, SK, TF, and HI. (2) Collection and assembly of data: TK, DU, TF, YI, TF. (3) Analysis and interpretation of the data: TK, DU, SO, SK, TF, TF, YI and YT. (4) Drafting of the article: TK, DU, SO, SK, TF, TF, YI, YT, and HI. (5) Critical revision of the article for important intellectual content: TK, DU, SO, SK, TF, and HI. (6) Final approval of the article: TK, DU, SO, SK, TF, TF, YI, YT, and HI. (7) Statistical expertise: TK, DU, SO, YT. (8) Obtaining financial support: DU, SO. 


\section{Acknowledgments}

We would like to thank Editage (www.editage.jp) for English language editing.

\section{References}

1. Mohd Sharif NA, Usman J, Wan Safwani WKZ, Siew Li G, Abdul Karim S, Mohamed NA, et al. Effects of simple knee sleeves on pain and knee adduction moment in early unilateral knee osteoarthritis. Proc Inst Mech Eng H. 2019;233:1132-40. https://doi.org/10.1177/0954411919874614.

2. Bennell KL, Bowles KA, Wang Y, Cicuttini F, Davies-Tuck M, Hinman RS. Higher dynamic medial knee load predicts greater cartilage loss over 12 months in medial knee osteoarthritis. Ann Rheum Dis. 2011;70:1770-4. https://doi.org/10.1136/ard.2010.147082.

3. Kainz H, Killen BA, Campenhout AV, Desloovere K, Aznar JMG, Shefelbine S, et al. ESB Clinical Biomechanics Award 2020: pelvis and hip movement strategies discriminate typical and pathological femoral growth - insights gained from a multi-scale mechanobiological modelling framework. Clin Biomech. 2021;87:1-9. https://doi.org/10.1016/j.clinbiomech.2021.105405.

4. Yoshimura N, Muraki S, Oka H, Mabuchi A, En-Yo Y, Yoshida M, et al. Prevalence of knee osteoarthritis, lumbar spondylosis, and osteoporosis in Japanese men and women: the research on osteoarthritis/osteoporosis against disability study. J Bone Miner Metab. 2009;27:620-8. https://doi.org/10.1007/s00774-009-0080-8.

5. Winby CR, Lloyd DG, Besier TF, Kirk TB. Muscle and external load contribution to knee joint contact loads during normal gait. J Biomech. 2009;42:2294-300. https://doi.org/10.1016/j.jbiomech.2009.06.019.

6. Sharma L, Hurwitz DE, Thonar EJ, Sum JA, Lenz ME, Dunlop DD, et al. Knee adduction moment, serum hyaluronan level, and disease severity in medial tibiofemoral osteoarthritis. Arthritis Rheum. 1998;41:1233-40. https://doi.org/10.1002/1529-0131(199807)41:7<1233::AID-ART14>3.0.C0;2-L.

7. Levinger P, Menz HB, Morrow AD, Bartlett JR, Feller JA, Bergman NR. Relationship between foot function and medial knee joint loading in people with medial compartment knee osteoarthritis. $J$ Foot Ankle Res. 2013;6:33. https://doi.org/10.1186/1757-1146-6-33.

8. Shih KS, Chien HL, Lu TW, Chang CF, Kuo CC. Gait changes in individuals with bilateral hallux valgus reduce first metatarsophalangeal loading but increase knee abductor moments. Gait Posture. 2014;40:38-42. https://doi.org/10.1016/j.gaitpost.2014.02.011.

9. Gross KD, Felson DT, Niu J, Hunter DJ, Guermazi A, Roemer FW, et al. Association of flat feet with knee pain and cartilage damage in older adults. Arthritis Care Res. 2011;63:937-44. https://doi.org/10.1002/acr.20431.

10. Uritani D, Fukumoto T, Myodo T, Fujikawa K, Usui M, Tatara D. The association between toe grip strength and osteoarthritis of the knee in Japanese women: A multicenter cross-sectional study. PLOS ONE. 2017;12:e0186454. https://doi.org/10.1371/journal.pone.0186454. 
11. Langley B, Cramp M, Morrison SC. Clinical measures of static foot posture do not agree. J Foot Ankle Res. 2016;9:1-6. https://doi: 10.1186/s13047-016-0180-3. eCollection.

12. Lerner ZF, DeMers MS, Delp SL, Browning RC. How tibiofemoral alignment and contact locations affect predictions of medial and lateral tibiofemoral contact forces. J Biomech. 2015;48:644-50. https://doi.org/10.1016/j.jbiomech.2014.12.049.

13. Holder J, Trinler U, Meurer A, Stief F. A systematic review of the associations between inverse dynamics and musculoskeletal modeling to investigate joint loading in a clinical environment. Front Bioeng Biotechnol. 2020;8:603907. https://doi.org/10.3389/fbioe.2020.603907.

14. Delp SL, Loan JP, Hoy MG, Zajac FE, Topp EL, Rosen JM. An interactive graphics-based model of the lower extremity to study orthopaedic surgical procedures. IEEE Trans. Biomed. Eng. 1990;37:757767.10.1109/10.102791

15. Lerner ZF, Board WJ, Browning RC. Pediatric obesity and walking duration increase medial tibiofemoral compartment contact forces. J Orthop Res. 2016;34:97-105. https://doi.org/10.1002/jor.23028.

16. Stief F, Feja Z, Holder J, van Drongelen S, Adolf S, Braun S, et al. Non-invasive determination of frontal plane lower limb alignment using motion capture technique - an alternative for full-length radiographs in young patients treated by a temporary hemiepiphysiodesis? Gait Posture. 2020;79:26-32. doi: 10.1016/j.gaitpost.2020.04.011.

17. McLaughlin P, Vaughan B, Shanahan J, Martin J, Linger G. Inexperienced examiners and the Foot Posture Index: A reliability study. Report 26. Man Ther. 2016;26:238-40. https://doi.org/10.1016/j.math.2016.06.009.

18. Maes R, Dojcinovic S, Andrianne Y, Burny F. Study of the plantar arch: correlations between podometrical and radiological parameters. Results of a prospective study of 79 cases. Rev Med Brux. 2006;27:422-9.

19. Abramoff MD, Magelhaes PJ, Ram SJ. Image processing with ImageJ, Biophoton Int. 2004;11:3642.

20. Janssen DM, Sanders AP, Guldemond NA, Hermus J, Walenkamp GH, Van Rhijn LW. A comparison of hallux valgus angles assessed with computerised plantar pressure measurements, clinical examination and radiography in patients with diabetes. J Foot Ankle Res. 2014;7:33. https://doi.org/10.1186/1757-1146-7-33.

21. Ohi $\mathrm{H}$, lijima $\mathrm{H}$, Fukutani $\mathrm{N}$, Aoyama $\mathrm{T}$, Kaneda $\mathrm{E}$, Ohi $\mathrm{K}$, et al. Varus thrust visualized during gait was associated with inverted foot in patients with knee osteoarthritis: an exploratory study. Gait Posture. 2018;61:269-75. https://doi.org/10.1016/j.gaitpost.2018.01.020.

22. Deng J, Joseph R, Wong CK. Reliability and validity of the sit-to-stand navicular drop test: do static measures of navicular height relate to the dynamic navicular motion during gait? J Stud Phys Ther Res. 2010;2:21-8.

23. Uritani $D$, Fukumoto $T$, Matsumoto $D$. Intrarater and interrater reliabilities for a toe grip dynamometer. J Phys Ther Sci. 2012;24:639-43. https://doi.org/10.1589/jpts.24.639. 
24. Gerus P, Sartori M, Besier TF, Fregly BJ, Delp SL, Banks SA, et al. Subject-specific knee joint geometry improves predictions of medial tibiofemoral contact forces. J Biomech. 2013;46:2778-86. https://www.sciencedirect.com/science/article/pii/S0021929013004144\#f0020.

25. Merireles S, Groote FD, Reeves ND, Verschueren S, Maganaris C, Luyten F, et al. Knee contact forces are not altered in early knee osteoarthritis, Gait Posture. 2016;45:115-20. https://www.sciencedirect.com/science/article/pii/S0966636216000175\#fig0010.

26. Kim W, Voloshin AS. Role of plantar fascia in the load bearing capacity of the human foot. J Biomech. 1995;28:1025-33. https://doi.org/10.1016/0021-9290(94)00163-x.

27. Byrnes SK, Wearing S, Böhm H, Dussa CU, Horstmann T. Effects of idiopathic flatfoot deformity on knee adduction moments during walking. Gait Posture. 2021;84:280-6. https://doi.org/10.1016/j.gaitpost.2020.12.021.

28. Haim A, Rozen N, Dekel S, Halperin N, Wolf A. Control of knee coronal plane moment via modulation of center of pressure: a prospective gait analysis study. J Biomech. 2008;41:3010-6. 10.1016/j.jbiomech.2008.07.029. jbiomech.2008.07.029.

29. Buldt AK, Forghany S, Landorf KB, Levinger P, Murley GS, Menz HB. Foot posture is associated with plantar pressure during gait: A comparison of normal, planus and cavus feet. Gait Posture. 2018;62:235-40. https://doi.org/10.1016/j.gaitpost.2018.03.005.

30. Levinger P, Menz HB, Morrow AD, Feller JA, Bartlett JR, Bergman NR. Foot kinematics in people with medial compartment knee osteoarthritis. Rheumatol (Oxf Engl). 2012;51:2191-8. doi: 10.1093/rheumatology/kes222.

31. Sanchis-Sales E, Sancho-Bru JL, Roda-Sales A, Pascual-Huerta J. Effect of static foot posture on the dynamic stiffness of foot joints during walking. Gait Posture. 2018;62:241-6.

32. Behling AV, Nigg BM. Relationships between the foot posture Index and static as well as dynamic rear foot and arch variables. J Biomech. 2020. https://doi:

10.1016/j.jbiomech.2019.109448;98:109448.

33. Nester $\mathrm{CJ}$, Hutchins $S$, Bowker P. Shank rotation: a measure of rearfoot motion during normal walking. Foot Ankle Int. 2000;21:578-83. doi: 10.1177/107110070002100709.

\section{Figures}




\section{Total CF}

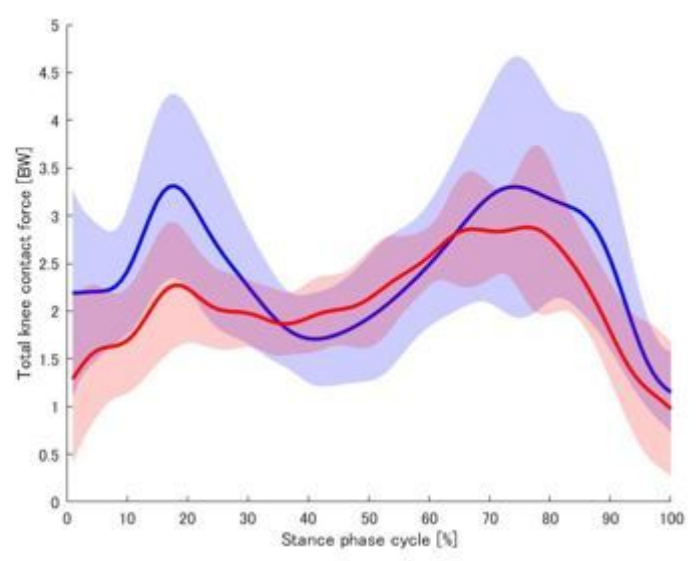

Medial CF

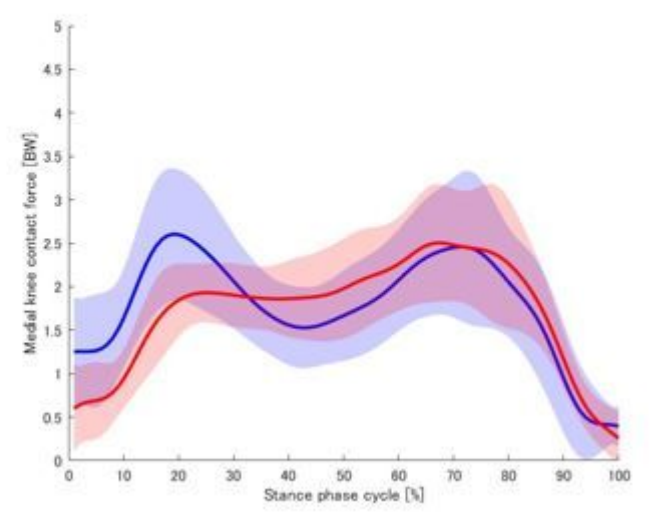

\section{Lateral CF}

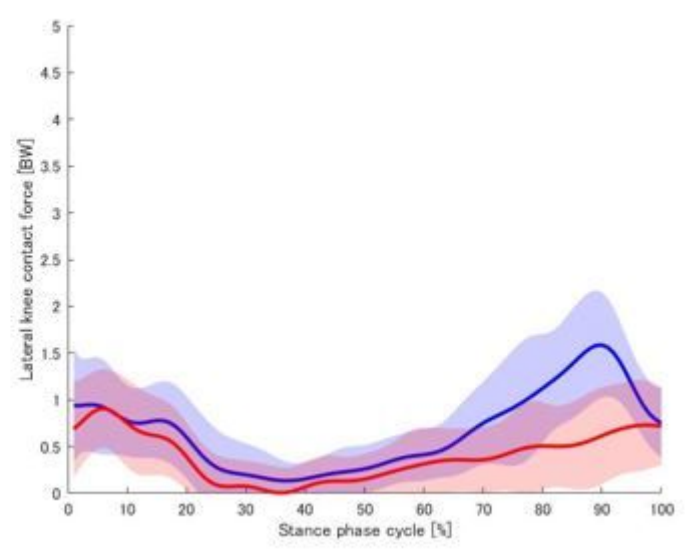

\section{Figure 1}

Force on the total, medial, and lateral tibiofemoral joint during the stance phase. Data are presented as mean \pm standard deviation. Controls and patients with knee OA are presented as blue and red lines, respectively. CF, Tibiofemoral contact force. 
Biceps femoris (lh)

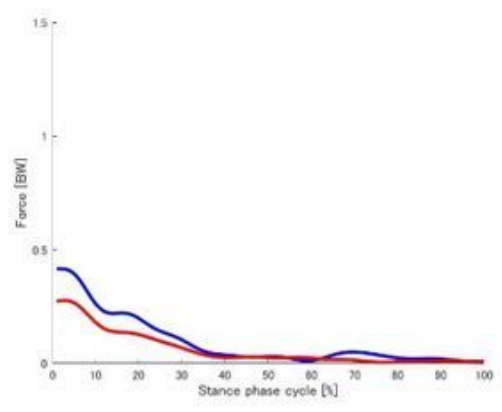

Semimembranosus

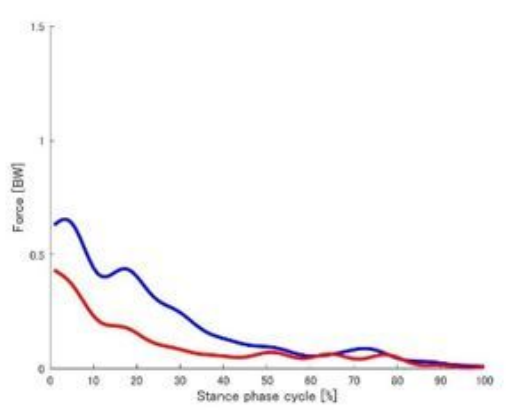

Vastus lateralis

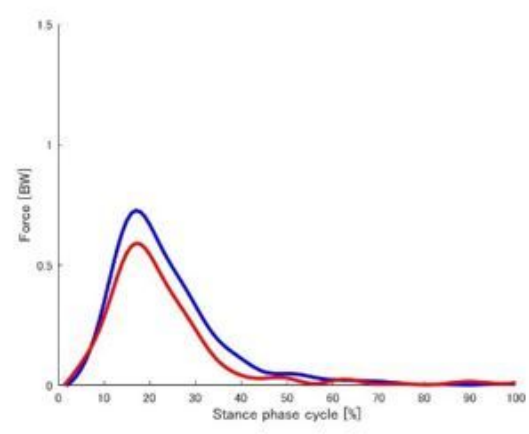

Biceps femoris (sh)

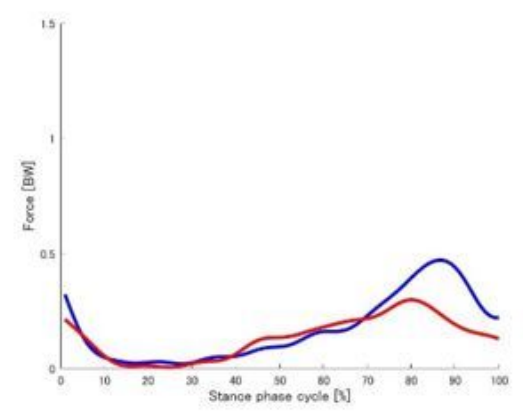

Rectus femoris

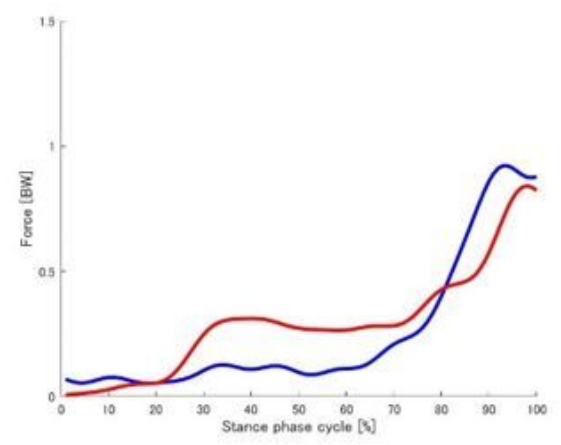

Gastrocnemius (med)

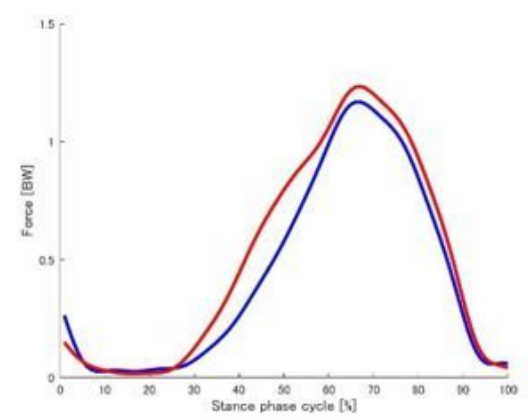

Semitendinosus

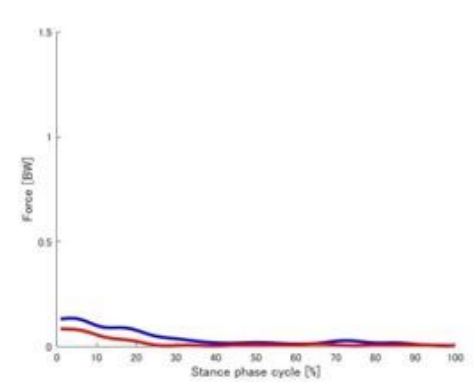

Vastus medialis

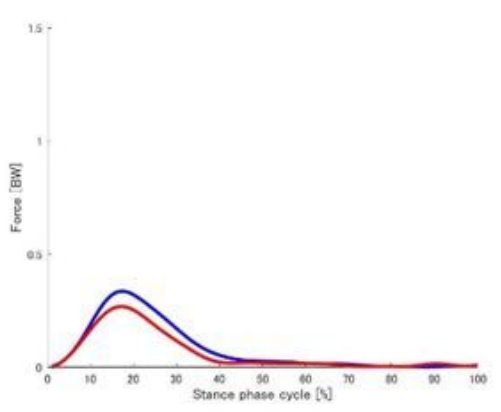

Gastrocnemius (lat)

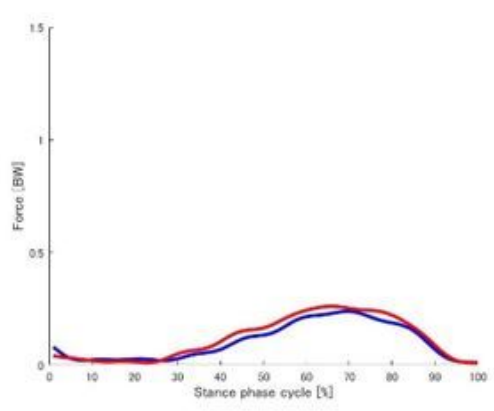

Figure 2

Muscle forces for knee-spanning muscles during the stance phase. Data are presented as mean \pm standard deviation. Controls and patients with knee $\mathrm{OA}$ are presented as blue and red lines, respectively. Lh, long head. Sh, short head. Med, medialis. Lat, lateralis. 


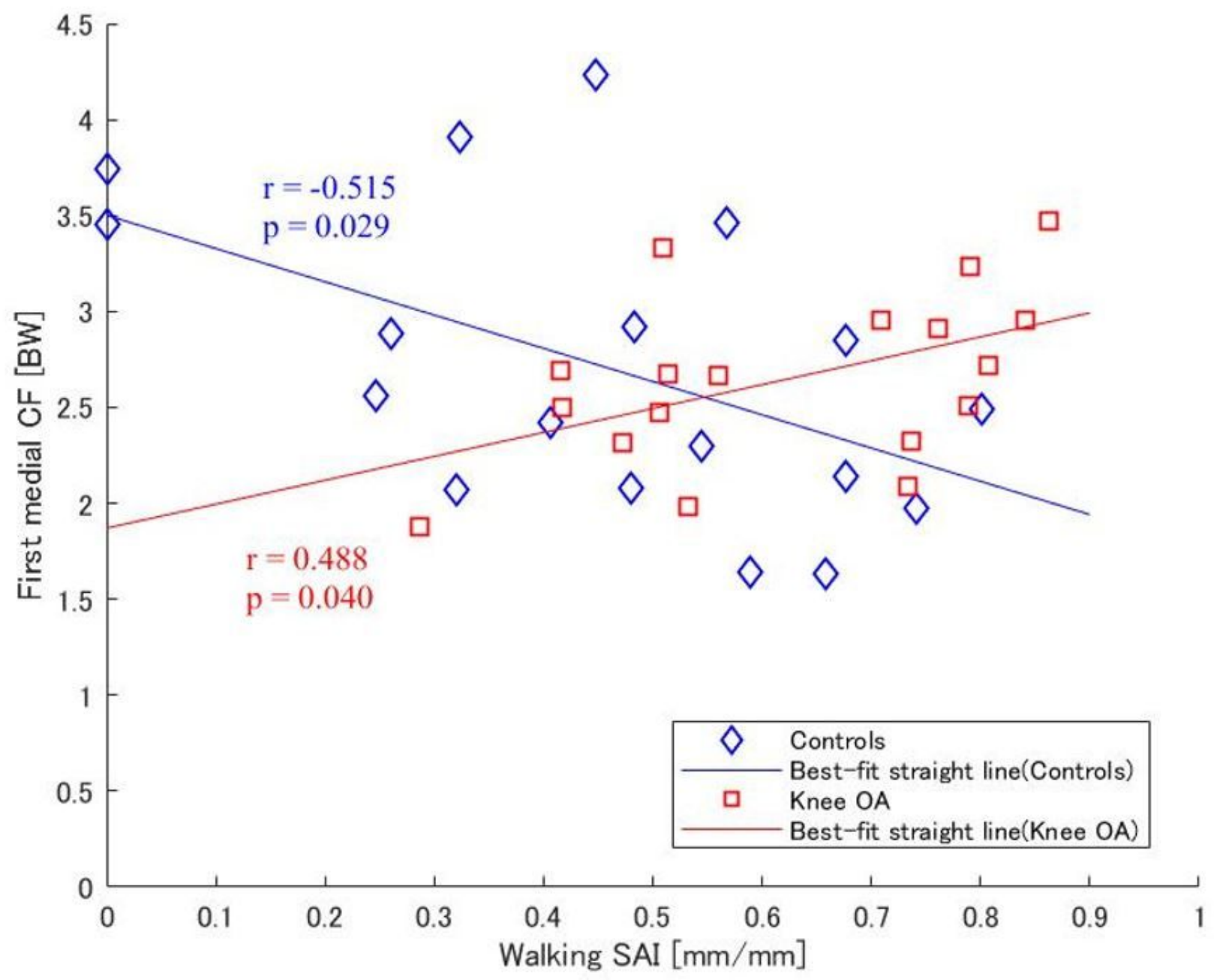

Figure 3

Scatter plot with the best fit line (solid line) for Pearson's correlations. The correlation analysis was performed between first medial CF and SAI for controls (diamonds) and knee OA (squares). CF, Tibiofemoral contact force. SAI, Staheli Arch Index.

\section{Supplementary Files}

This is a list of supplementary files associated with this preprint. Click to download.

- DataSet.xlsx 